Supporting Information:

\title{
Temperature and Pressure Dependence of Salt-Brine Dihedral Angles in the Subsurface
}

\author{
Jessica M. Rimsza ${ }^{1, *}$ and Kristopher L. Kuhlman ${ }^{2}$
}

1. Geochemistry Department, Sandia National Laboratories, Albuquerque, NM 87123

2. Applied Systems Analysis \& Research, Sandia National Laboratories, Albuquerque, NM 87123

*Corresponding author: jirimsza@,sandia.gov

Table S1. Interaction Potential Parameters* 20

\begin{tabular}{|c|c|c|c|}
\hline L-J Interaction & $\varepsilon_{i i}(\mathrm{kcal} / \mathrm{mol})$ & $\sigma_{i i}(\AA)$ & $q_{i}(\mathrm{e})$ \\
\hline $\mathrm{O}-\mathrm{O}$ & 0.1553 & 3.1660 & -0.8476 \\
\hline $\mathrm{H}-\mathrm{H}$ & - & - & 0.4238 \\
\hline $\mathrm{Na}^{+}-\mathrm{Na}^{+}$ & 0.3526 & 2.1595 & 1.0000 \\
\hline $\mathrm{K}^{+}-\mathrm{K}^{+}$ & 0.4297 & 3.1860 & 1.0000 \\
\hline $\mathrm{Cl}^{--}$ & 0.0128 & 4.8305 & -1.0000 \\
\hline
\end{tabular}

${ }^{*} q_{i}$ is charge and $\varepsilon_{i i}$ and $\sigma_{i i}$ are the L-J parameters

Table S2: Variable molarity of $\mathrm{NaCl}$ and $\mathrm{KCl}$ brines in contact with a $\mathrm{NaCl}$ surface. No pressure dependence on molarity occurred.

\begin{tabular}{|c|c|c|c|}
\hline \multirow{2}{*}{ Temperature (K) } & \multirow{2}{*}{ Pressure (MPa) } & \multicolumn{2}{|c|}{ Total Concentration (m) } \\
\cline { 3 - 4 } & & $\mathrm{NaCl}$ & $\mathrm{KCl}$ \\
\hline 300 & $0-150$ & 6.19 & 4.80 \\
\hline 350 & $0-150$ & 6.41 & 6.47 \\
\hline 400 & $0-150$ & 6.92 & 7.96 \\
\hline 450 & $0-150$ & 7.64 & 9.54 \\
\hline
\end{tabular}

Table S3: Variable molality of $\mathrm{NaCl}+\mathrm{KCl}$ brines in contact with a $\mathrm{NaCl}$ surface. No pressure dependence on molality occurred.

\begin{tabular}{|c|c|c|c|c|}
\hline \multirow{2}{*}{ Temperature (K) } & \multirow{2}{*}{ Pressure (MPa) } & \multicolumn{3}{|c|}{ Partial Molality (m) } \\
\cline { 3 - 5 } & & $\mathrm{Na}$ & $\mathrm{K}$ & $\mathrm{Cl}$ \\
\hline 300 & $0-150$ & 4.54 & 3.96 & 8.49 \\
\hline 350 & $0-150$ & 4.63 & 4.05 & 8.67 \\
\hline 400 & $0-150$ & 4.61 & 5.78 & 10.39 \\
\hline 450 & $0-150$ & 4.83 & 7.31 & 12.09 \\
\hline
\end{tabular}

Table S4: Change in shear modulus (GPa) with temperature (K) and pressure (GPa) 


\begin{tabular}{|c|c|c|c|c|c|c|c|c|}
\hline \multicolumn{2}{|c|}{} & 0 & 25 & 50 & 75 & 100 & 125 & 150 \\
\hline \multirow{4}{*}{$\begin{array}{c}\text { Temperature } \\
(\text { K) }\end{array}$} & 300 & 12.61 & 12.62 & 12.63 & 12.64 & 12.67 & 12.70 & 12.73 \\
\cline { 2 - 9 } & 325 & 12.52 & 12.53 & 12.54 & 12.55 & 12.58 & 12.61 & 12.64 \\
\cline { 2 - 9 } & 350 & 12.43 & 12.44 & 12.45 & 12.47 & 12.49 & 12.52 & 12.55 \\
\cline { 2 - 9 } & 400 & 12.26 & 12.26 & 12.28 & 12.29 & 12.32 & 12.34 & 12.38 \\
\cline { 2 - 9 } & 450 & 12.09 & 12.09 & 12.10 & 12.12 & 12.14 & 12.17 & 12.20 \\
\hline
\end{tabular}

Table S5: Change in lattice constant $(\AA)$ with temperature $(\mathrm{K})$ and pressure $(\mathrm{GPa})$

\begin{tabular}{|c|c|c|c|c|c|c|c|c|}
\hline & \multicolumn{7}{|c|}{ Pressure (MPa) } \\
\hline & & 0 & 25 & 50 & 75 & 100 & 125 & 150 \\
\hline \multirow{5}{*}{$\begin{array}{c}\text { Temperature } \\
\text { (K) }\end{array}$} & 300 & 5.64 & 5.64 & 5.64 & 5.63 & 5.63 & 5.62 & 5.62 \\
\hline & 325 & 5.64 & 5.64 & 5.64 & 5.63 & 5.63 & 5.63 & 5.62 \\
\hline & 350 & 5.64 & 5.64 & 5.64 & 5.64 & 5.63 & 5.63 & 5.62 \\
\hline & 400 & 5.64 & 5.64 & 5.64 & 5.64 & 5.63 & 5.63 & 5.62 \\
\hline & 450 & 5.65 & 5.65 & 5.64 & 5.64 & 5.64 & 5.63 & 5.63 \\
\hline
\end{tabular}

Table S6: Change in the solid-solid interfacial tension $(\mathrm{N} / \mathrm{m})$ with temperature $(\mathrm{K})$ and pressure (GPa)

\begin{tabular}{|c|c|c|c|c|c|c|c|c|}
\hline & \multicolumn{7}{|c|}{ Pressure (MPa) } \\
\hline & & 0 & 25 & 50 & 75 & 100 & 125 & 150 \\
\hline \multirow{5}{*}{$\begin{array}{c}\text { Temperature } \\
(\mathrm{K})\end{array}$} & 300 & 0.73 & 0.73 & 0.73 & 0.73 & 0.73 & 0.73 & 0.73 \\
\hline & 325 & 0.72 & 0.72 & 0.72 & 0.72 & 0.72 & 0.72 & 0.73 \\
\hline & 350 & 0.72 & 0.72 & 0.72 & 0.72 & 0.72 & 0.72 & 0.72 \\
\hline & 400 & 0.71 & 0.71 & 0.71 & 0.71 & 0.71 & 0.71 & 0.71 \\
\hline & 450 & 0.70 & 0.70 & 0.70 & 0.70 & 0.70 & 0.70 & 0.70 \\
\hline
\end{tabular}

Table S7: Change in dihedral angle as a function of brine composition and concentration for $\mathrm{NaCl}, \mathrm{KCl}$, and $\mathrm{NaCl}+\mathrm{KCl}$ brines within the $300-450 \mathrm{~K}$ temperature range and $0-150 \mathrm{MPa}$ pressure range. Experimental data from Reference: ${ }^{45}$

\begin{tabular}{|l|c|c|c|c|c|c|c|}
\hline & \multicolumn{2}{|c|}{$\mathrm{NaCl}$} & \multicolumn{2}{c|}{$\mathrm{KCl}$} & \multicolumn{2}{c|}{ Mixed } & \multirow{2}{*}{ Expt. } \\
\cline { 2 - 8 } & $5.0 \mathrm{M}$ & $\mathrm{Var}$ & $5.0 \mathrm{M}$ & $\mathrm{Var}$ & $5.0 \mathrm{M}$ & Var & \\
\hline Dihedral Angle Range $\left(^{\circ}\right)$ & $55-72$ & $65-74$ & $60-76$ & $72-78$ & $54-74$ & $76-85$ & $55-72$ \\
\hline
\end{tabular}

